\title{
On Bistable Region of Slotted-ALOHA Systems with Delay Constraints
}

\author{
Takayuki Horiuchi, Katsumi Sakakibara*, Jumpei Taketsugu \\ Department of Information and Communication Engineering, Okayama Prefectural University, Soja, 719-1197, Japan
}

Copyright @2016 by authors, all rights reserved. Authors agree that this article remains permanently

open access under the terms of the Creative Commons Attribution License 4.0 International License

\begin{abstract}
Bistable behavior of slotted ALOHA systems has been extensively investigated. It is proved that bistable region can be eliminated by limiting the number of allowable transmission attempts per packet to eight or less. In this paper, we investigate bistable behavior of slotted ALOHA systems with delay constraints in terms of the catastrophe theory. A lifetime of a packet is confined within $D$ slots, that is, a packet will be discarded after timer of $D$ time-slots expired, unless packet transmission succeeds. Letting $r$ be the packet transmission probability in a slot, we indicate that there exists bistable region, if the product $D r$ is greater than approximately 8.30, and that the system can operate with the unique stable equilibrium point, if $\mathrm{Dr}$ is less than approximately 8.30 .
\end{abstract}

Keywords Slotted ALOHA, Stability, Catastrophe Theory, Bistable Region, Delay Constraints

\section{Introduction}

Bistable behavior of random access protocols has been extensively investigated, since 1970 s [1, 2]. It has been widely recognized that bistable behavior of random access protocols may suddenly deteriorate the performance of the system due to the unexpected slip into an undesired stable operating point [3]. Carleial and Hellman analyzed the stability of ALOHAtype protocols in terms of the expected drift [1]. The system possesses two stable operating points, if it exhibits bistable behavior; one of which offers comparatively high throughput, whereas the other produces considerably low throughput [2]. To make matters worse, the system operating at the "good and desired" stable point may suddenly slip into a catastrophic "air pocket," which contingently occurs as a result of an unexpected avalanche of offered load. In order to mitigate undesired bistable behavior of random access protocols, backoff algorithms and retransmission cutoff are effective countermeasures $[4,5,6]$. From another point of view, it has been widely and conventionally implemented in random access protocols that retransmissions of a packet are given up after the corresponding timer expires [7].

The catastrophe theory [8] is another method to analyze stability of random access protocols [9, 10, 11, 12, 13], which can succeed in explicitly indicating bistable region. Onozato et al. [10] proved that there exists the cusp catastrophe in slotted ALOHA systems. Yokohira et al. [11] applied the catastrophe theory to the analysis of CSMA/CD (Carrier Sense Multiple Access with Collision Detection) and indicated the existence of the cusp catastrophe. Fantacci et al. [13] discussed the stability of PRMA (Packet Reservation Multiple Access). Extending the results in [10], Sakakibara et al. proved that slotted ALOHA system can operate with the unique stable equilibrium point, if the number of retransmission trials is limited to at most eight [14, 15]. Tyagi et al. [16] discussed the conditions on the offered load which results in bistable operation in slotted ALOHA systems.

In this paper, we investigate bistable region of slotted ALOHA systems with delay constraints in terms of the catastrophe theory and explicitly indicate the bistable region. Under the delay constraints, a lifetime of a packet is confined within $D$ time-slots. Assuming that a node transmits a packet in its buffer with probability $r$, we show that there exists a cusp catastrophe in the system if the product $D r$ is approximately greater than 8.30 , and that the system is mono-stable if $\mathrm{Dr}$ is less than 8.30 .

The rest of the present paper is organized as follows: Section 2 presents a system model of slotted ALOHA with delay constraints. In Section 3, bistable behavior is analyzed in terms of the catastrophe theory, particularly, the cusp catastrophe. Numerical results and discussions are presented in Section 4. Finally, Section 5 concludes the present paper.

\section{System Model}

We consider a slotted ALOHA system with $N$ singlebuffer users, which contend a shared common channel consisting of time-slots of unit length $[1,2,10,14,15]$. A user with empty buffer generates a new packet of unit length with probability $\lambda$ at the beginning of each time slot. It follows from the single-buffer assumption that a user with occupied buffer generates no new packets. A lifetime of a packet is confined within $D$ time-slots, so that a packet in the buffer will be discarded after the timer of $D$ time-slots expires. A packet in the buffer is transmitted with probability $r$ at each time-slot as long as the timer is running. A packet transmis- 


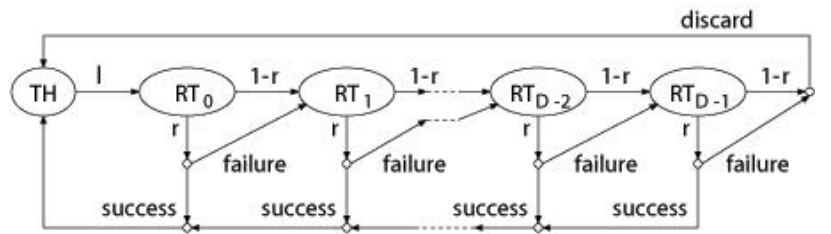

Figure 1. Markovian model with respect to user's state.

sion succeeds if and only if no other packets are transmitted simultaneously. No packet errors on the channel and no capture effect are taken into account.

According to the assumptions above, we can construct a Markovian model with respect to the user's state, as shown in Fig. 1. In Fig. 1, State TH represents a user with empty buffer and State $\mathrm{RT}_{d}$ corresponds to a user with a packet after the elapse of $d$ time-slots. A user in State TH generates a new packet with probability $\lambda$ and moves to State $\mathrm{RT}_{0}$. A user in State $\mathrm{RT}_{d}$ transmits its packet with probability $r$. It returns to State $\mathrm{TH}$ if the packet transmission succeeds. Otherwise, it transits to $\mathrm{RT}_{d+1}$ for $d=0,1, \ldots, D-2$, if it fails in packet transmission or if it abandons packet transmission with probability $1-r$. A packet of a user in State $\mathrm{RT}_{D-1}$ will be dropped, unless its transmission succeeds. Note here that each user exists in one of State $\mathrm{TH}$ and $\mathrm{RT}_{d}$ for $d=0,1, \ldots, D-1$ in every time-slot.

\section{Analysis}

\subsection{Equations in Equilibrium}

Let $n_{d}$ denote the average number of users in State $\mathrm{RT}_{d}$ for $d=0,1, \ldots, D-1$. Then, the average number of backlogged users is given by

$$
n=\sum_{d=0}^{D-1} n_{d}
$$

and that of users in State TH is $N-n$. The offered load to the common channel is evaluated as

$$
G=n r \leq N r .
$$

Next, we derive expressions for the average increment of users in each state in Fig. 1. Using the Poisson approximation for the offered load $G$, the probability of successful transmission $P_{\text {succ }}$ is

$$
P_{\text {succ }}=(1-r)^{n-1} \simeq e^{-G},
$$

since no simultaneous transmissions result in successful transmission.

The in-flow to State TH consists of a user with successful transmission and users with packet dropping. The average increment of users in State TH is

$$
\begin{aligned}
\delta_{\mathrm{TH}}= & n r P_{\mathrm{succ}}+n_{D-1}(1-r)+n_{D-1} r\left(1-P_{\mathrm{succ}}\right) \\
& \quad-(N-n) \lambda \\
\simeq & G e^{-G}+n_{D-1}\left(1-r e^{-G}\right)-(N-n) \lambda .
\end{aligned}
$$

Similarly, the average increment of users in State $\mathrm{RT}_{d}$ can be evaluated as

$$
\delta_{0}=(N-n) \lambda-n_{0}
$$

and

$$
\begin{aligned}
\delta_{d} & =n_{d-1} r\left(1-P_{\mathrm{succ}}\right)+n_{d-1}(1-r)-n_{d} \\
& =n_{d-1}\left(1-r e^{-G}\right)-n_{d}
\end{aligned}
$$

for $d=1,2, \ldots, D-1$.

In equilibrium, the in-flow equals to the out-flow at each state. Thus, the relation $\delta_{\mathrm{TH}}=\delta_{0}=\delta_{1}=\cdots=\delta_{D-1}=0$ holds.

\subsection{Balance Function}

From (5) and (6), we can solve $\delta_{0}=\delta_{1}=\cdots=\delta_{D-1}=0$ recursively and obtain

$$
n_{d}=\frac{N \lambda\left\{1-\left(1-r e^{-G}\right)^{D}\right\}}{r e^{-G}+\lambda\left\{1-\left(1-r e^{-G}\right)^{D}\right\}}
$$

for $d=0,1, \ldots, D-1$. By substituting (7) into (1), the average number of backlogged users in equilibrium is

$$
n=\frac{1-\left(1-r e^{-G}\right)^{D-1}}{r e^{-G}} .
$$

We substitute (7) and (8) into (4). Then, the offered load $G$ in equilibrium can be obtained as a root of the following equation:

$$
\begin{aligned}
& A(G \mid N \lambda, N r, r, D) \\
& =G e^{-G}-\frac{N \lambda N r e^{-G}\left\{1-\left(1-r e^{-G}\right)^{D}\right\}}{N r e^{-G}+N \lambda\left\{1-\left(1-r e^{-G}\right)^{D}\right\}} \\
& =0,
\end{aligned}
$$

where $A(G \mid N \lambda, N r, r, D)$ is referred to as the balance function $[14,15]$. We can easily examine that

$$
\begin{aligned}
A(0 \mid N \lambda, N r, r, D) & =-\frac{N \lambda N r\left\{1-(1-r)^{D}\right\}}{N r+N \lambda\left\{1-(1-r)^{D}\right\}} \\
& <0
\end{aligned}
$$

and

$$
\begin{aligned}
& A(N r \mid N \lambda, N r, r, D) \\
& =\frac{(N r)^{2} e^{-N r}}{N r e^{-N r}+N \lambda\left\{1-\left(1-r e^{-N r}\right)^{D}\right\}} \\
& >0
\end{aligned}
$$

for given $N, \lambda, r$ and $D$. It implies that there exists at least one positive root $G$ in the range of $(0, N r)$ for (9). Slotted ALOHA systems is mono-stable, if (9) has a unique positive root $G$. The system is bistable, if (9) has more than one positive $\operatorname{root} G$.

\subsection{Cusp Catastrophe and Bifurcation Sets}

Following the catastrophe theory [8], there exists the cusp catastrophe in the slotted ALOHA system with delay constraints, if the system of equations

$$
\left\{\begin{array}{l}
A(G \mid N \lambda, N r, r, D)=\frac{\partial A}{\partial G}=\frac{\partial^{2} A}{\partial G^{2}}=0 \\
\frac{\partial^{3} A}{\partial G^{3}} \neq 0
\end{array}\right.
$$

has roots $(G, N \lambda, N r)$ for given transmission probability $r$ and delay limit $D$, where $G<N, 0<\lambda \leq 1$ and $0<r \leq 1$. Note that the catastrophe theory is briefly reviewed in Appendix. When the cusp catastrophe exists, the cusp point is given by 
the root of (12) and the bifurcation sets, $B^{+}$and $B^{-}$, are defined as

$$
\left\{\begin{array}{c}
B^{+}=\left\{(N \lambda, N r) \mid A(G \mid N \lambda, N r, r, D)=\frac{\partial A}{\partial G}=0,\right. \\
\left.\frac{\partial^{2} A}{\partial G^{2}}>0, N \lambda>0, N r>0\right\} \\
B^{-}=\left\{(N \lambda, N r) \mid A(G \mid N \lambda, N r, r, D)=\frac{\partial A}{\partial G}=0,\right. \\
\left.\frac{\partial^{2} A}{\partial G^{2}}<0, N \lambda>0, N r>0\right\}
\end{array}\right.
$$

Here, we examine the bifurcation sets obtained from $A(G$ $N \lambda, N r, r, D)=\partial A / \partial G=0$.

It follows from $A(G \mid N \lambda, N r, r, D)=0$ that

$$
N \lambda=\frac{N r G e^{-G}}{(N r-G)\left\{1-\left(1-r e^{-G}\right)^{D}\right\}} .
$$

Differentiating the balance function $A(G \mid N \lambda, N r, r, D)$ with respect to $G$, we can obtain

$$
\begin{aligned}
\frac{\partial A}{\partial G}= & N r(1-G) e^{-G} \\
& +N \lambda\left\{1-\left(1-r e^{-G}\right)^{D}\right\} \\
& -D N \lambda(G-N r) r e^{-G}\left(1-r e^{-G}\right)^{D-1}
\end{aligned}
$$

After substituting (14) into (15) and solving $\partial A / \partial G=0$ with respect to $N r$, we have

$$
N r=\frac{G^{2} f(G, r, D)}{h(G, r, D)},
$$

where

$$
\begin{aligned}
f(G, r, D)= & \left\{1-\left(1-r e^{-G}\right)^{D}\right\} \\
& -D r e^{-G}\left(1-r e^{-G}\right)^{D-1} \\
h(G, r, D)= & (G-1)\left\{1-\left(1-r e^{-G}\right)^{D}\right\} \\
& -D r G e^{-G}\left(1-r e^{-G}\right)^{D-1}
\end{aligned}
$$

Notice here that (14) and (16) for $r=1.0$ are identical to (7) and (8) in [14], respectively. Therefore, the slotted ALOHA system with delay constraints for $r=1.0$ is mono-stable, if the delay limit $D$ is less than or equal to eight, and that there exists the bistable region in the system, if $D \geq 9$.

In order for (14) and (16) to be the valid bifurcation sets, both $N \lambda$ and $N r$ should be positive.

First, since $G \leq N r$ from (2), it is clear that $N \lambda>0$.

Second, consider the sign of $N r$ defined by (16), which depends on the sign of the functions $f(G, r, D)$ and $h(G, r, D)$. Since from (17) we have

$$
\begin{aligned}
\frac{\partial f}{\partial G}= & -D_{r} e^{-G}\left(1-r e^{-G}\right)^{D-1} \\
& -D(D-1) r^{2} e^{-2 G}\left(1-r e^{-G}\right)^{D-2}<0,
\end{aligned}
$$

$f(G, r, D)$ is a decreasing function with respect to $G$. In addition, it can be shown that $\lim _{G \rightarrow \infty} f(G, r, D)=0$. As a result, it is proved that $f(G, r, D)>0$ for any $G>0$.

Next, we examine the sign of $h(G, r, D)$ in a similar manner to the proof of the existence of the fold catastrophe in (8)

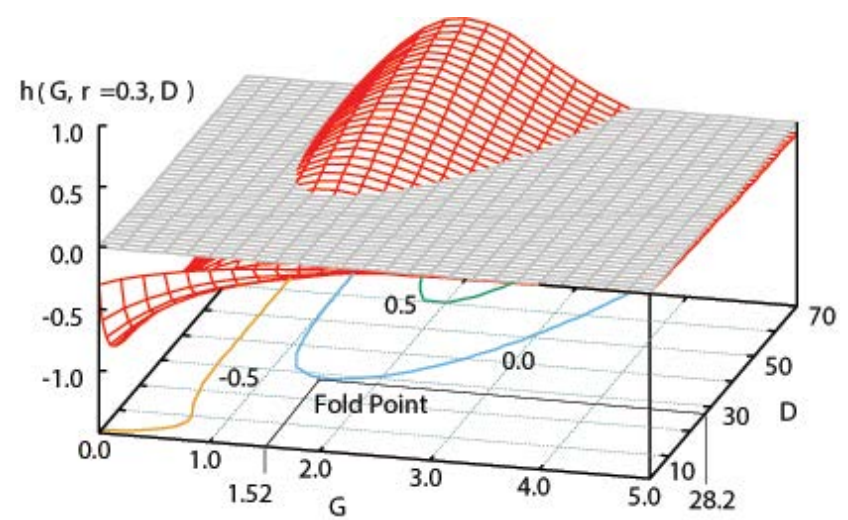

Figure 2. Function $h(G, r=0.3, D)$.

in [14]. From (18) we have

$$
\begin{aligned}
\frac{\partial h}{\partial G}= & 1-\left(1-r e^{-G}\right)^{D} \\
& -D(D-1) G r^{2} e^{-2 G}\left(1-r e^{-G}\right)^{D-2} \\
\frac{\partial^{2} h}{\partial G^{2}}= & -D r e^{-G}\left(1-r e^{-G}\right)^{D-1} \\
& +D(D-1)(2 G-1) r^{2} e^{-2 G}\left(1-r e^{-G}\right)^{D-2} \\
& -D(D-1)(D-2) G r^{3} e^{-3 G}\left(1-r e^{-G}\right)^{D-3}
\end{aligned}
$$

For given $r$, we can solve $h(G, r, D)=\partial h / \partial G=0$ numerically with respect to $(G, D)$. Furthermore, we can numerically check $\partial^{2} h / \partial G^{2} \neq 0$ for these values of $G, D$ and $r$, as discussed in Subsection 4.2. Therefore, there exists the fold catastrophe in $h(G, r, D)$.

For example, behavior of $h(G, r, D)$ for $r=0.3$ is illustrated in Fig. 2. As shown in Fig. 2, the fold point of the function $h(G, r=0.3, D)$ is $(G, D) \approx(1.52,28.2)$. Hence, if $D<28.2$, then it follows that $h(G, r=0.3, D)<0$ for any values of offered load $G$, which implies that $N r<0$ from (16), so that no valid bifurcation sets exit. In consequence, the slotted ALOHA with delay constraints for $r=0.3$ is mono-stable for delay limit $D \leq 28$ and bistable for $D \geq 29$.

\section{Numerical Results and Discussions}

\subsection{Bistable Region}

Bistable region for various given delay limit $D$ is shown in Fig. 3. The horizontal axis $N \lambda$ is the average traffic when no backlogged users exist and the vertical axis $N r$ is the maximum of the average traffic when all the users are backlogged, that is, when there are no users with empty buffer. Red and green solid lines represent bifurcation sets $B^{+}$and $B^{-}$for $D r=9.0$ and $D r=15.0$, respectively, and blue dashed lines are bifurcation sets for $r=1.0$. For $r=1.0$, a backlogged user transmits the packet in every time-slot. Furthermore, the results in [14] address that a slotted ALOHA system is bistable, if more than eight transmission trials are permitted. Therefore, the bifurcation sets for $r=1.0$, blue dashed lines, are identical to the bifurcation sets shown in [14].

In Fig. 3, for given $N r$, the slotted ALOHA system possesses two stable equilibrium operating points, if $N \lambda$ is between $B^{+}$and $B^{-}$. Otherwise, the system can operate with the unique globally stable equilibrium point. Mono-stable region with light (resp. heavy) traffic is referred to as the active region (resp. saturated region). If the system operates in 


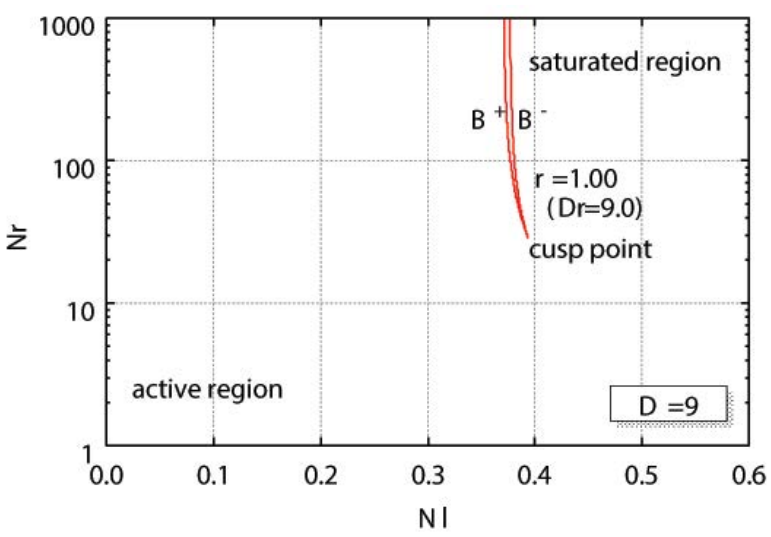

(a) For delay limit $D=9$ (slots)

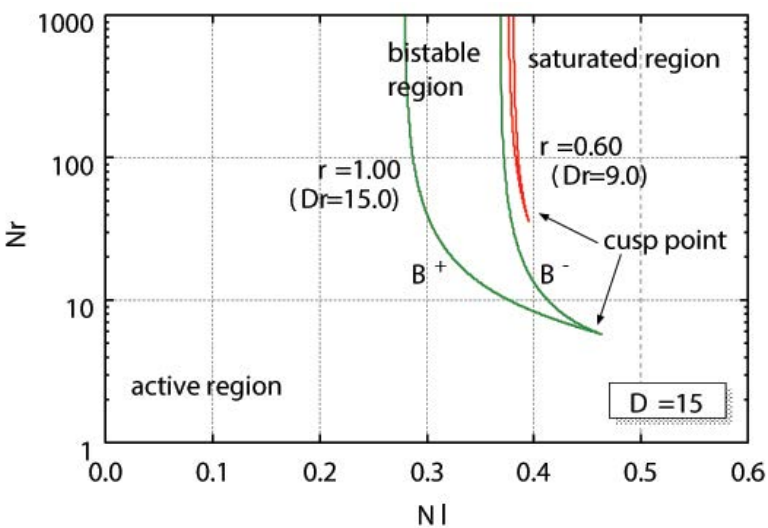

(c) For delay limit $D=15$ (slots)

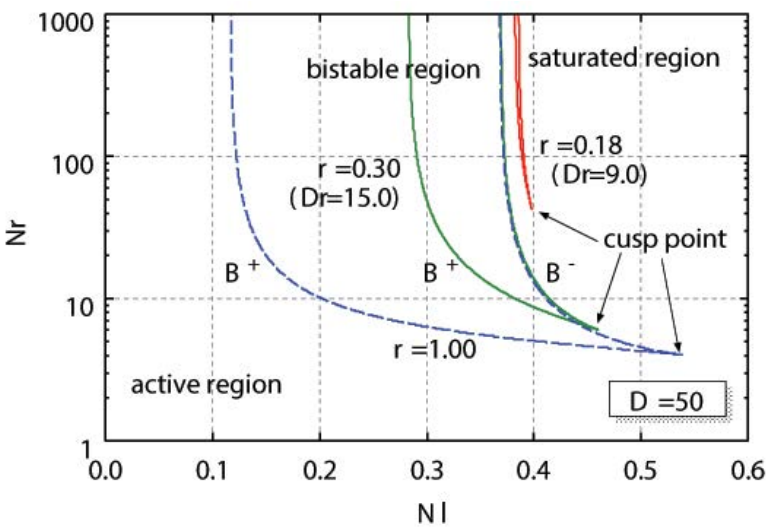

(e) For delay limit $D=50$ (slots)

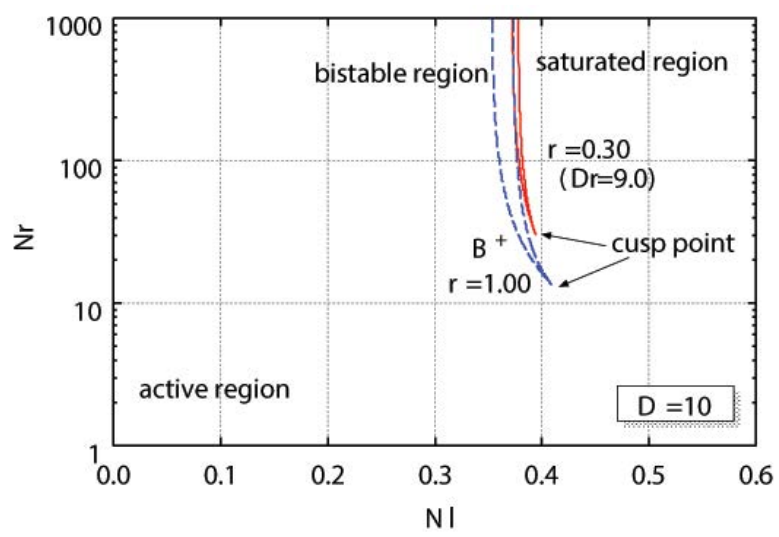

(b) For delay limit $D=10$ (slots)

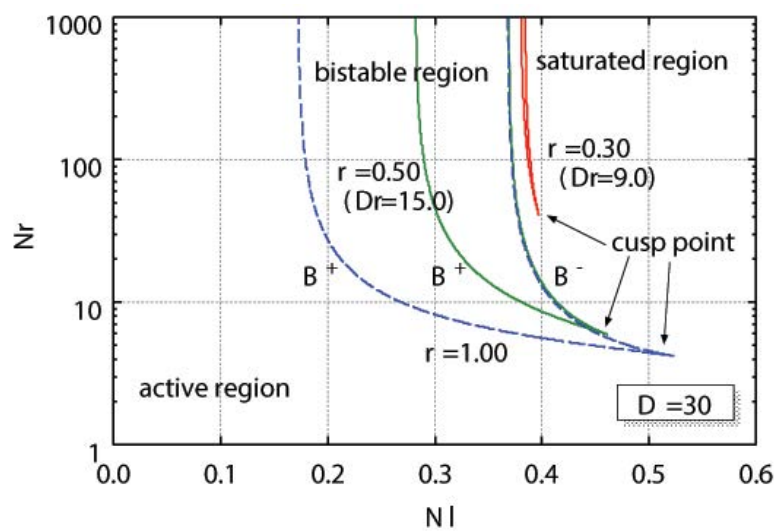

(d) For delay limit $D=30$ (slots)

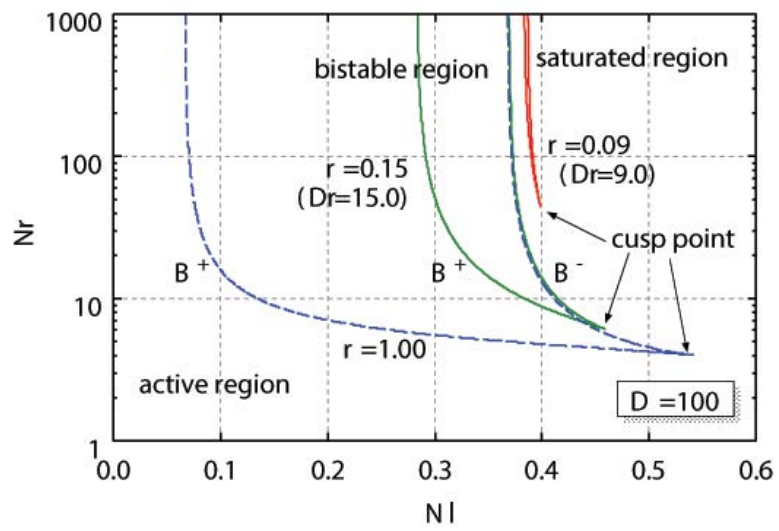

(f) For delay limit $D=100$ (slots)

Figure 3. Bistable region of slotted ALOHA systems with delay constraints for $D r=9.0$ (red solid lines), $D r=15.0$ (green solid lines) and $r=1.0$ (blue dashed lines).

the active region, there exists a unique globally stable equilibrium point which provides comparatively moderate traffic and high throughput. The system also possesses a unique globally stable equilibrium point even if it operates in the saturated region. However, the system suffers from heavy traffic in contrast and low throughput can be achieved. It can be observed that bistable region is unchanging for given $\mathrm{Dr}$ and that how bistable region grows according to increment of $D$. Similarly to Fig. 3 in [14], one of the bifurcation sets, $B^{-}$, is stable with respect to an increment of delay limit $D$, whereas the other, $B^{+}$, moves to smaller $N \lambda$. Therefore, the bistable region expands by curtailing the active region rather than the saturated region.

We can observe from Fig. 3 that bifurcation sets $B^{+}$and $B^{-}$ may converge to a certain non-trivial constant when $N r$ goes to infinity. For given delay limit $D$ and packet transmission probability $r$, we have from (14)

$$
\lim _{N r \rightarrow \infty} N \lambda=\frac{G e^{-G}}{1-\left(1-r e^{-G}\right)^{D}},
$$

where $G$ is the offered load for $N r \rightarrow \infty$. Since it follows from (16) that $N r$ goes to infinity when $h(G, r, D)=0$, the offered load $G$ in (22) can be evaluated as a root of $h(G, r, D)=0$ for given $D$ and $r$,

Asymptotic values of bifurcation sets $B^{+}$and $B^{-}$for $\mathrm{Nr} \rightarrow$ $\infty$ are illustrated in Fig. 4 for $r=0.09$ (red solid lines), $r=$ 0.3 (green solid lines), and $r=1.0$ (blue dashed lines). In Fig. 4, for example, bifurcation sets appear for $r=0.3$, if delay limit $D$ is greater than 28.2, as shown in Fig. 2. We can find that the shape of the three curves are almost identical and 


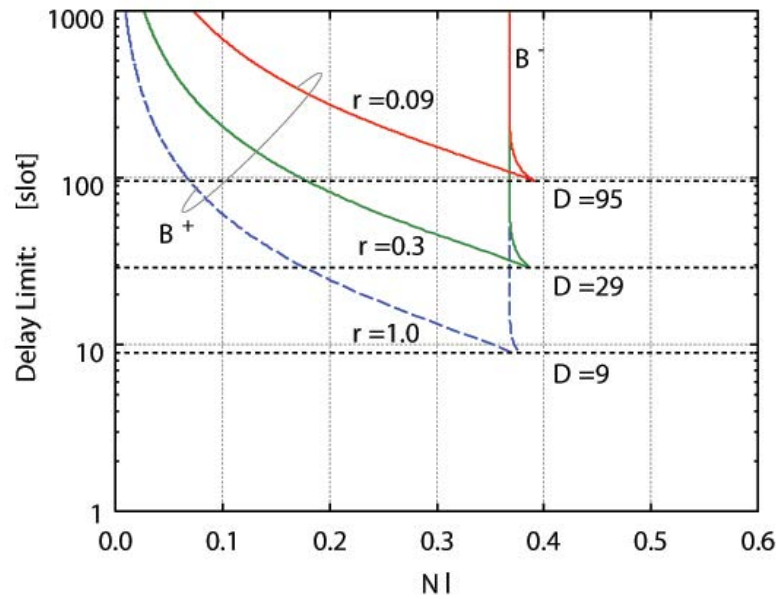

Figure 4. Asymptotic values of bifurcation sets $B^{+}$and $B^{-}$for $N r \rightarrow \infty$ and for $r=0.09$ (red solid lines), $r=0.3$ (green solid lines) and $r=1.0$ (blue dashed lines).

they move up with preservation of approximately $\mathrm{Dr}=8.30$ according to decrement of $r$.

\subsection{Trajectory of Fold Points in $h(G, r, D)$}

As indicated in the previous subsection, for given packet transmission probability $r$, there may exist the threshold of delay limit $D$, above which the bistable region appears in the slotted ALOHA system with delay constraints. The threshold can be obtained from the fold point of $h(G, r, D)$. Here, let us examine the trajectory of the fold points in $h(G, r, D)$; particularly the dependency of $D$ on $r$.

First, we show the trajectory of $G$ and $D$ for given $r$ in Fig. 5 and Fig. 6, respectively, which can be obtained by numerically solving $h(G, r, D)=\partial h / \partial G=0$ for given $r$. The second derivative $\partial^{2} h / \partial G^{2}$ evaluated at the root of $h(G, r, D)=\partial h / \partial G=0$ is also depicted in Fig. 5 by blue solid line. We can see that $\partial^{2} h / \partial G^{2} \neq 0$. As a result, it is confirmed that roots of $h(G, r, D)=\partial h / \partial G=0$ provide the fold points.

Next, let us scrutinize the dependency of $D$ on $r$ at fold points, shown in Fig. 6, where a general view is shown in Fig. 6(a) and an enlarged view for $0.290 \leq r \leq 0.310$ is depicted in Fig. 6(b). Using (18) and (20), we can obtain

$$
r=\frac{e^{G}}{(G-1)(D-1)+1}
$$

from $h(G, r, D)=\partial h / \partial G=0$. Since it follows from (18) that $h(G, r, D)<0$ for $G<1$, the value of $G$ at the fold points is greater than one, for example, as shown in Fig. 5. It results in

$$
\frac{\partial r}{\partial D}=-\frac{e^{G}(G-1)}{\{(G-1)(D-1)+1\}^{2}}<0
$$

at the fold points, so that the packet transmission probability $r$ is a decreasing function of delay limit $D$. Further, as aforementioned, delay limit $D$ at the fold point for $r=1.0$ is approximately 8.30 [14].

Since the number of transmissions allowed within delay limit $D$ time-slots is subject to the binomial distribution with probability $r$, the average of the maximum number of transmissions within $D$ time-slots is $D r$. Therefore, a hyperbolic curve of $D r=8.30$ is also shown by blue dashed line in

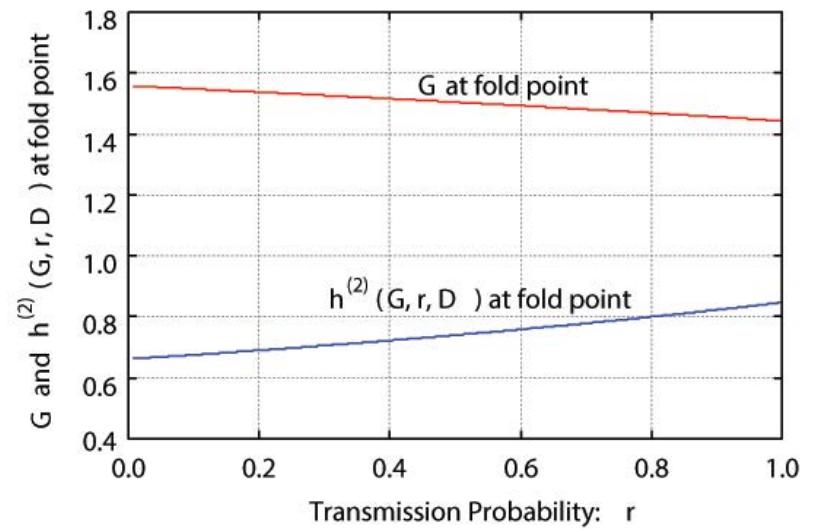

Figure 5. Trajectory of $G$ (red solid line) and $\partial^{2} h / \partial G^{2}$ (blue solid line) for given $r$.

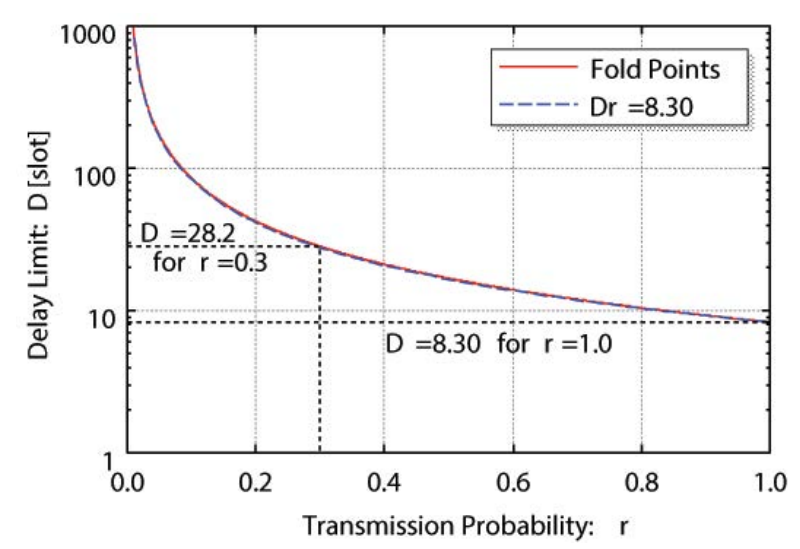

(a) General view

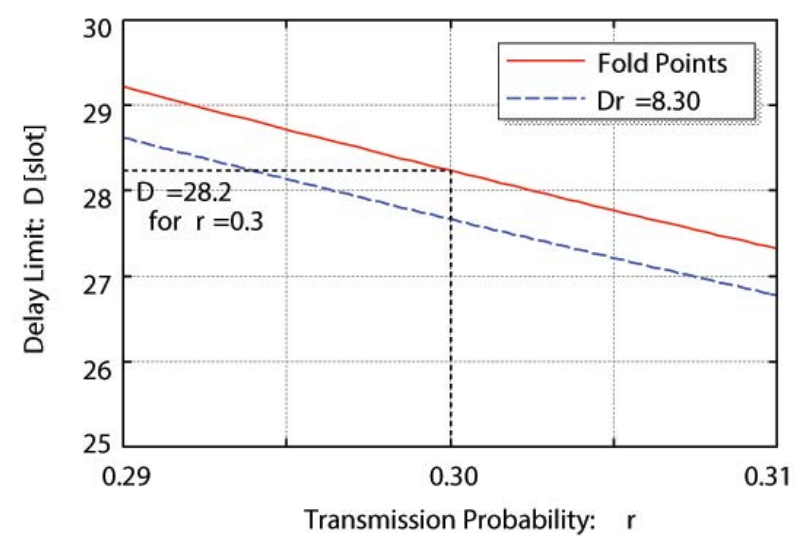

(b) Enlarged view for $0.29 \leq r \leq 0.31$

Figure 6. Trajectory of $D$ (red solid line) and hyperbolic curve $\mathrm{Dr}=8.30$ (blue dashed line) for given $r$.

Fig. 6 for reference. We can observe in Fig. 6(a) good agreement between two curves, although an enlarged view reveals slight differences between exact results and approximation by the hyperbolic curve, as shown in Fig. 6(b). For instance, the value of $D$ at the fold point for $r=0.3$ is 28.2, as shown in Fig. 2, while the corresponding hyperbolic curve provides $D \approx 8.30 / 0.3=27.7$.

From the above observations, the slotted ALOHA system with delay constraints can operate mono-stably, if the product of delay limit $D$ and packet transmission probability $r$ is approximately less than 8.30. On the contrary, there exists the bistable region if the product $\mathrm{Dr}$ is greater than 8.30. 
Table 1. Thom's seven elementary catastrophes [8]

\begin{tabular}{ll|l|l}
\hline$k$ & $v$ & \multicolumn{1}{|c}{ typical potential function $P(x, c)$} & \multicolumn{1}{|c}{ name } \\
\hline 1 & 1 & $x^{3}+c x$ & fold \\
2 & 1 & $x^{4}+c_{1} x^{2}+c_{2} x$ & cusp \\
3 & 1 & $x^{5}+c_{1} x^{3}+c_{2} x^{2}+c_{3} x$ & swallowtail \\
3 & 2 & $x_{1}^{3}+x_{2}^{3}+c_{1} x_{1} x_{2}+c_{2} x_{1}+c_{3} x_{2}$ & hyperbolic umbilic \\
3 & 2 & $x_{1}^{3}+x_{1} x_{2}^{2}+c_{1}\left(x_{1}^{2}+x_{2}^{2}\right)+c_{2} x_{1}+c_{3} x_{2}$ & elliptic umbilic \\
4 & 1 & $x^{6}+c_{1} x^{4}+c_{2} x^{3}+c_{3} x^{2}+c_{4} x$ & butterfly \\
4 & 2 & $x_{1}^{2} x_{2}+x_{2}^{4}+c_{1} x_{1}^{2}+c_{2} x_{2}^{2}+c_{3} x_{1}+c_{4} x_{2}$ & parabolic umbilic \\
\hline
\end{tabular}

\section{Conclusion}

In this paper, we have investigated bistable behavior of slotted ALOHA systems with delay constraints in terms of the catastrophe theory and explicitly indicated the bistable region. Under the delay constraints, a lifetime of a packet is confined within $D$ time-slots. Assuming that a node transmits a packet in its buffer with probability $r$ in a time-slot, we have shown that the slotted ALOHA system with delay constraints has two stable equilibrium points if the product $\mathrm{Dr}$ is approximately greater than 8.30 , and that the system is mono-stable if $\mathrm{Dr}$ is less than 8.30. In addition, we have shown that bistable region basically depends on the product $D r$, not on individual parameters $D$ and $r$.

\section{Appendix}

In this appendix, we briefly review the catastrophe theory established by Thom [8]. For more detailed description, see $[8,9,12]$.

Suppose a dynamic system governed by a set of $k$ control parameters. Let $P(x, c): \mathbb{R}^{v} \times \mathbb{R}^{k} \longrightarrow \mathbb{R}$ be the potential function of the system, where $v$ is the dimension of the state space of the system, $x \in \mathbb{R}^{v}$ is the state space of the system, and $c \in \mathbb{R}^{k}$ is a set of $k$ control parameters. The critical points for which $\partial P(x, c) / \partial x=0$ are called equilibrium points. An equilibrium point is stable if $\partial^{2} P(x, c) / \partial x^{2}>0$ and unstable if $\partial^{2} P(x, c) / \partial x^{2}<0$. An equilibrium point where $\partial^{2} P(x, c) / \partial x^{2}=0$ is called a bifurcation point. The drift or the balance function is defined as $\partial P(x, c) / \partial x$, and its sign indicates the inverse direction of system evolution from point $x$. The catastrophe theory is concerned with equilibrium points that arise when the potential function changes.

The potential functions can be classified according to their shape or surface of equilibrium points, which are essentially determined by the number of control parameters. Thom's list of seven elementary catastrophes is presented in Table 1 .

In this paper, two elementary catastrophes are considered, the cusp and the fold catastrophes. For given $D$ and $r$, $A(G \mid N \lambda, N r, r, D)$ in (9) can be viewed as the derivative of a certain potential function with two control parameters $N \lambda$ and $N r ; k=2$, and one-dimensional state space $G \in \mathbb{R}^{1}$; $v=1$. If $A(G \mid N \lambda, N r, r, D)<0$, the system is likely to evolve so as to increase the offered load $G$. On the other hand, the system tends to lessen $G$ if $A(G \mid N \lambda, N r, r, D)>0$. Thus, there may exist the cusp or the fold catastrophe. In terms of the cusp catastrophe, the critical point for which $\partial P(x, c) / \partial x=\partial^{2} P(x, c) / \partial x^{2}=\partial^{3} P(x, c) / \partial x^{3}=0$; or equivalently (12), is referred to as the cusp point and a set of bifurcation points, as the bifurcation set; as shown in (13).
In addition, for given $r, h(G, r, D)$ in (18) can be regarded as the derivative of another potential function with one control parameter $D ; k=1$, and one-dimensional state space $G$; $v=1$. Thus, there may exist the fold catastrophe. In terms of the fold catastrophe, a bifurcation point is referred to as the fold point.

\section{Acknowledgment}

A part of this work was supported by Japan Society for the Promotion of Science (JSPS) KAKENHI Grant Number 16K06363.

\section{REFERENCES}

[1] A. B. Carleial and M. E. Hellman, Bistable behavior of ALOHA-type systems. IEEE Trans. Commun., Vol. COM-23, No. 4, pp. 401-410, 1975.

[2] Y. C. Jenq, On the stability of slotted ALOHA systems. IEEE Trans. Commun., Vol. COM-28, No. 11, pp. 1936-1939, 1980.

[3] G. E. Keiser, Local Area Networks, McGraw-Hill, New York City, NY, 1989.

[4] J. Goodman, A. G. Greenberg, N. Madras and P. March, Stability of binary exponential backoff, J. ACM, Vol. 35, No. 3, pp. 579-602, 1988.

[5] G. Bianchi, Performance analysis of the IEEE 802.11 distributed coordination function, IEEE J. Select. Areas Commun., Vol. 18, No. 3, pp. 535-547, 2000.

[6] J. H. Sarker and S. J. Halme, An optimum retransmission cutoff scheme for slotted ALOHA, Wireless Personal Commun., Vol. 13, No. 1-2, pp. 185-202, 2000.

[7] E. Hossain and V. K. Bhargava, On higher layer protocol performance in CDMA S-ALOHA networks with packet combining in Rayleigh fading channels, in Proc. IEEE GLOBECOM 2000, San Francisco, CA, Nov.-Dec. 2000.

[8] R. Thom, Structural Stability and Morphogenesis: An Outline of a General Theory of Models, W. A. Benjamin, Reading, MA, 1975.

[9] N. D. Birrell, Application of catastrophe theory to a slotted ALOHA communication system, IEE Proc.-F, Vol. 130, No. 4, pp. 337-342, 1983.

[10] Y. Onozato and S. Noguchi, On the thrashing cusp in slotted ALOHA systems, IEEE Trans. Commun., Vol. COM-33, No. 11, pp. 1171-1182, 1985. 
[11] T. Yokohira, T. Nishida and H. Miyahara, Analysis of dynamic behavior in p-persistent CSMA/CD using cusp catastrophe, Comput. Networks \& ISDN Sys., Vol. 12, No. 5, pp. 277-289, 1986.

[12] R. Nelson, Stochastic catastrophe theory in computer performance modeling, J. ACM, Vol. 34, No. 3, pp. 661-685, 1987.

[13] R. Fantacci and F. Innocenti, Performance evaluation of a modified PRMA protocol for joint voice and data packet wireless networks, IEEE Trans. Commun., Vol. 47, No. 12, pp. 1837-1848, 1999.

[14] K. Sakakibara, H. Muta and Y. Yuba, The effect of limiting the number of retransmission trials on the stability of slotted
ALOHA systems, IEEE Trans. Veh. Tech., Vol. 49, No. 4, pp. 1449-1453, 2000.

[15] K. Sakakibara and J. Taketsugu, Bistable region of backoff algorithms with contention window in slotted ALOHA systems, Cyber J.: Multidisciplinary J. Sci. \& Tech., J. Select. Areas Telecommun., Vol. 2012, No. 4, pp. 9-14, 2012.

[16] R. R. Tyagi, F. Aurzada, K.-D. Lee, S. G. Kim and M. Reisslein, Impact of retransmission limit on preamble contention in LTE-Advanced network, IEEE Systems J., Vol.9, No. 3, pp. 752-765, 2015. 